\title{
Fault Prediction in Fuzzy Discrete Event Systems: A Diagnoser Approach
}

\author{
Bilal Benmessahel \\ Department of Basic Studies in Technology, Faculty of Technology \\ University of Sétif 1, 19000 Sétif, Algeria \\ bilal.benmessahel@gmail.com

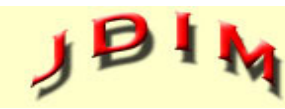 \\ Journal of Digital \\ Information Management \\ Farid Nouioua \\ Computer Science Department, Mohamed El Bachir El Ibrahimi University, Bordj Bou Arreridj \\ Algeria \\ farid.nouioua@gmail.com \\ Mohamed Touahria \\ Computer Science Department, University of Ferhat Abbas Setif 1, Maabouda City \\ Algeria \\ mohamed.touahria@univ-setif.dz \\ Abderrahim Chariete \\ Auto-entrepreneur R \& D Informatique, 4 rue de Vienne, 90000, Belfort \\ France \\ charieteabderrahim@hotmail.com
}

\begin{abstract}
In this workm we study the fault prediction in fuzzy discrete event systems. Fuzzy discrete event systems are proposed to deal with vagueness, impreciseness, and subjectivity in real-world problems. The verification is divided into two steps. In the first step, we give a method to construct a Diagnoser. And in the second step, based on the structure of diagnoser we give the necessary and sufficient conditions to verify the future occurrence of the fault. The newly proposed approach allows us to deal with the problem of fault prediction for both crisp DESs and FDESs. Finally, an example is provided to illustrate the efficiency of the proposed approach.
\end{abstract}

Subject Categories and Descriptors: [I.2.3 Deduction and Theorem Proving]; Uncertainty, "fuzzy," and probabilistic reasoning: [B.1.3 Control Structure Reliability, Testing, and Fault-Tolerance]

General Terms: Fault Prediction, Fuzzy Models, Discrete Events

Keywords: Fuzzy Discrte Systems, Fault Prediction

Received: 18 April 2019, Revised 27 July 2019, Accepted 10 August 2019
Review Metrics: Review Scale- 0/6, Review Score-4.95, Interreviewer Consistency- 86\%

DOI: $10.6025 / \mathrm{jdim} / 2019 / 17 / 6 / 337-345$

\section{Introduction}

Today the probability that a possible fault occurs in technological systems is more and more greater. Moreover, some faults can cause very serious accidents causing economic or human losses.

Fault prediction is the task that deals with the study of the possible occurring of future event in the system based on the observation of events. The Fault prediction in the framework of fuzzy discrete event systems (FDESs) has not received enough attention since most of the recent research of fault prediction in the literature dealt with crisp discrete event systems. In this work, we attempt to fill this gap and we address the problem of fault prediction in FDESs where we give a new approach called "Diagnoser approach".

The paper is organized as follows. In section 2, the fuzzy 
discrete-event systems (FDESs) model is presented. In Section 3, we give the verification approach and the diagnoser construction. Section 4 the necessary and sufficient condition for Fault prediction in FDESs using diagnoser are given. In Section 5, we provide an illustrative example. Finally, section 6 concludes the paper.

\section{Fuzzy Discrete-event Systems}

Fuzzy discrete-event systems (FDESs) combine fuzzy set theory [1] with crisp DESs [2]. They have been successfully applied to many real-world complex systems such as biomedical systems in which vagueness, impreciseness, and subjectivity are typical features.

In this work, we use FDES model with fuzzy observability which has been proposed in [3] and [4]. In this framework, FDESs are modeled as fuzzy automata with fuzzy states and fuzzy events denoted by vectors and matrices, respectively. If the crisp state set is $Q=\left\{q_{1}, q_{2}, \ldots, q_{n}\right\}$ then a fuzzy state $\tilde{q}$ is written as a vector $\left[a_{1}, a_{2}, \ldots, a_{n}\right]$, where $a_{i} \in[0,1]$ represents the possibility of the current state being $q_{i}$. Similarly, a fuzzy event $\tilde{\sigma}$ is a matrix $\left[a_{i j}\right]_{n \times n}$, where each $a_{i j} \in[0,1]$ represents the possibility of the system transiting from state $q_{i}$ to state $q_{j}$ when $\widetilde{\sigma}$ occurs. Formally, fuzzy discrete-event systems are defined as follows.

Definition 1 [5] A fuzzy automaton is a system $\widetilde{G}=(\widetilde{Q}, \widetilde{E}$, $\tilde{\delta}, \tilde{q}_{0}$ ), where $\widetilde{Q}$ is a set of fuzzy states, $\tilde{q}_{0}$ is the initial fuzzy state and $\tilde{E}$ is the set of fuzzy events; the transition function $\widetilde{\delta}: \widetilde{\mathrm{Q}} \times \widetilde{\mathrm{E}} \rightarrow \widetilde{\mathrm{Q}}$ is defined as $\widetilde{\delta}(\widetilde{\mathrm{q}}, \widetilde{\boldsymbol{\sigma}})=\tilde{\mathrm{q}} \odot \widetilde{\sigma}$. Note that $\odot$ is a max-min operation: for a matrix $A\left[a_{i j}\right]_{n \times m}$ and a matrix $B\left[b_{i j}\right]_{m \times k}, A \odot B=\left[c_{i j}\right]_{n \times k}$ is defined by:

$$
c_{i j}=\max _{l=1}^{m} \min \left\{a_{i l} c_{l j}\right\}
$$

\subsection{Prediction in Fuzzy DES}

In Fuzzy DESs, each fuzzy event has simultaneously membership in the observable event set, in the unobservable event set, and in the failure event set; with different degrees. We denote three fuzzy subsets: the unobservable event fuzzy subset: $\tilde{\Sigma}_{u o}: \tilde{E} \rightarrow[0,1]$, the observable event fuzzy subset $\tilde{\Sigma}_{0}: \tilde{E} \rightarrow[0,1]$ and the failure event fuzzy subset $\tilde{\Sigma}_{f}: \tilde{E} \rightarrow[0,1]$. Intuitively, $\tilde{\Sigma}_{u 0}(\tilde{\sigma})+\tilde{\Sigma}_{0}(\tilde{\sigma})=1$ and $\tilde{\Sigma}_{f}(\tilde{\sigma})$ describes the possibility of failure occurring on $\tilde{\sigma} \in \tilde{E}$ and $\tilde{\Sigma}_{o}(\tilde{\sigma})$ represent the observability degree of $\tilde{\sigma}$.

The language generated by $\widetilde{G}$, denoted as $L_{\tilde{G}}$ (or simply $L$ when it is clear from the context), is defined as follows:

$$
L=\left\{\widetilde{s} \in \widetilde{E}^{*}:(\exists \widetilde{q} \in \widetilde{Q}) \widetilde{\delta}\left(\widetilde{q}_{0}, \widetilde{s}\right)=\widetilde{q}\right\}
$$

$\tilde{\Sigma}_{f}(\tilde{s})=\max \left\{\tilde{\Sigma}_{f}(\tilde{\sigma}): \tilde{\sigma} \in \tilde{s}\right\}$ and $\tilde{\Sigma}_{o}(\tilde{s})=\min \left\{\tilde{\Sigma}_{o}(\tilde{\sigma}): \tilde{\sigma} \in \tilde{s}\right\} \operatorname{rep}-$ resent the failure degree and observability degree of the string $\tilde{s} \in L_{\tilde{G}}$, respectively.

We use the maximal observable event set $\widetilde{E}_{m o}$ as in [3]
[4] to avoid the case that the event set of the diagnoser constructed later is null. The set $\widetilde{E}_{m o}$ is composed of the events with the largest observability degree, i.e.

$$
\widetilde{E}_{m o}=\left\{\widetilde{\sigma} \in \widetilde{E}:\left(\forall \widetilde{\sigma}^{\prime} \in \widetilde{E}\right) \widetilde{\Sigma}_{\mathrm{o}}(\widetilde{\sigma}) \geq \widetilde{\Sigma}_{\mathrm{o}}\left(\widetilde{\sigma}^{\prime}\right)\right\}
$$

As usually, let $\tilde{G}$ be an FDES. We suppose that the laguage of $\widetilde{G}$ is live and $\widetilde{G}$ does not contain a cycle in which states are connected with unobservable events only. The post language of $L$ after $\widetilde{s}$ is the set of continuations of $\widetilde{s}$ in $\widetilde{G}$, i.e.

$$
L / \widetilde{s}=\left\{\widetilde{t} \in \widetilde{E}^{*}(\exists \widetilde{q} \in \widetilde{Q}) \widetilde{\delta}\left(\widetilde{q}_{0}, \tilde{s} \widetilde{t}\right)=\widetilde{q}\right\}
$$

Definition 2 [4] Let $\tilde{\sigma} \in \widetilde{E f}$, the $\widetilde{\sigma}$ - projection operation $\widetilde{\Pi}_{\tilde{\sigma}}: \widetilde{E}^{*} \rightarrow \widetilde{E}^{*}$ is defined as: $\widetilde{\Pi}_{\tilde{\sigma}}(\in)=\in$, and $\widetilde{\Pi}_{\tilde{\sigma}}(\widetilde{s} \widetilde{a})=\widetilde{\Pi}_{\widetilde{\sigma}}(s)$ $\widetilde{\Pi}_{\tilde{\sigma}}(\widetilde{a})$ for $\tilde{a} \in \tilde{E}$ and $\widetilde{s} \in \widetilde{E}$, where

$$
\widetilde{\Pi}_{\tilde{\sigma}}(a)=\left(\begin{array}{l}
\tilde{a}, \text { if } \tilde{a} \in \widetilde{E}_{m o} \text { or } \tilde{\Sigma}_{o}(\widetilde{a})>\tilde{\Sigma}_{o}(\widetilde{\sigma}) \\
\in, \text { otherwise }
\end{array}\right.
$$

The inverse projection operation is given by

$$
\tilde{\Pi}_{\tilde{\sigma}}^{-1}=\left\{\widetilde{s} \in \widetilde{E}^{*}(\exists \tilde{q} \in \widetilde{Q}) \tilde{\delta}\left(\widetilde{q}_{0}, \widetilde{s}\right)=\widetilde{q} \wedge \widetilde{\Pi}_{\tilde{\sigma}}(\widetilde{s})=\widetilde{t}\right\}
$$

The aim of $\tilde{\sigma}$ - projection is to erase the fuzzy events whose observability is not larger than $\widetilde{\Sigma}_{o}(\widetilde{\sigma})$.

In order to make a correct prediction decision, we specify an upper bound $\widetilde{\Sigma}_{f}(\widetilde{\sigma})$ for each $\tilde{\sigma} \in \widetilde{E}$. If the possibility of the failure occurring on a string $\tilde{s}$ exceeds the upper bound (i.e., $\widetilde{\Sigma}_{f}(\widetilde{s})>\widetilde{\Sigma}_{f}(\widetilde{\sigma})$ ) then we consider that $\widetilde{s}$ is a failure string. In the following, we formalize an approach of "fuzzy predictability" to predict the occurrence of those failure strings that exceed the specified upper bound.

We denote the set of faulty events by $\tilde{E}_{f}=\left\{\tilde{\sigma} \in \widetilde{E}_{i} \tilde{\Sigma}_{f}(\widetilde{\sigma})>0\right\}$. For $\tilde{\sigma} \in \widetilde{E}_{f}$, the set of all traces that end with an event whose possibility of failure occurring is not less than $\tilde{\Sigma}_{f}$ $(\widetilde{\sigma})$ is defined as follow:

$$
\widetilde{\Psi}_{\widetilde{\sigma}}\left(\tilde{\Sigma}_{f}\right)=\left\{\tilde{s} \in L: l_{\tilde{s}} \in \tilde{E}_{f}\right\}
$$

where $l_{\widetilde{s}}$ denotes the last event of $\widetilde{s}$.

Example 1 In $\tilde{G}=\left(\tilde{Q}, \tilde{E}, \tilde{\delta}, \tilde{q}_{0}\right)$ the set of fuzzy states is $\tilde{Q}=\left\{\tilde{q}_{0}, \tilde{q}_{1}, \tilde{q}_{2}, \tilde{q}_{3}, \tilde{q}_{4}, \tilde{q}_{5}\right\}$ and the set of fuzzy events $\tilde{E}=\{\tilde{\alpha}, \widetilde{\beta}, \tilde{\gamma}, \tilde{\theta}, \tilde{\tau}\}$. Each state in $\tilde{G}$ is denoted by a vector meaning $\tilde{q}=\left[a_{0}, a_{1}, a_{2}\right]$ that the system can simultaneously belong to three crisp states with membership degrees $a_{0}, a_{1}$ and $a_{2}$, respectively. Furthermore, a fuzzy event is represented by a $3 \times 3$ matrix.

$$
\tilde{\alpha}=\left(\begin{array}{lll}
\alpha_{11} & \alpha_{12} & \alpha_{13} \\
\alpha_{21} & \alpha_{22} & \alpha_{23} \\
\alpha_{31} & \alpha_{32} & \alpha_{33}
\end{array}\right)
$$

For instance, the fuzzy event $\alpha_{32} \in[0,1]$ represents the fact that the system transits from the third crisp state to 
the second crisp state with membership degree $\alpha_{32} \cdot \widetilde{q}_{0}=$ $\left[a_{0}, a_{1}, a_{2}\right]$ is the initial state. Then, we can calculate the other states by the max-min operation. Here, we omit the status describing the states and events. The observable and fault membership degrees of the fuzzy events are given as follows:

$$
\begin{aligned}
& \tilde{\Sigma}_{o}(\tilde{\alpha})=1, \tilde{\Sigma}_{o}(\tilde{\beta})=0.8, \tilde{\Sigma}_{o}(\tilde{\gamma})=0.6, \tilde{\Sigma}_{o}(\tilde{\theta})=0.1, \tilde{\Sigma}_{o}(\tilde{\tau})=0 \\
& \tilde{\Sigma}_{f}(\tilde{\alpha})=0, \tilde{\Sigma}_{f}(\tilde{\beta})=0.2, \tilde{\Sigma}_{f}(\tilde{\gamma})=0.3, \tilde{\Sigma}_{f}(\tilde{\theta})=0.7, \tilde{\Sigma}_{f}(\tilde{\tau})=0
\end{aligned}
$$

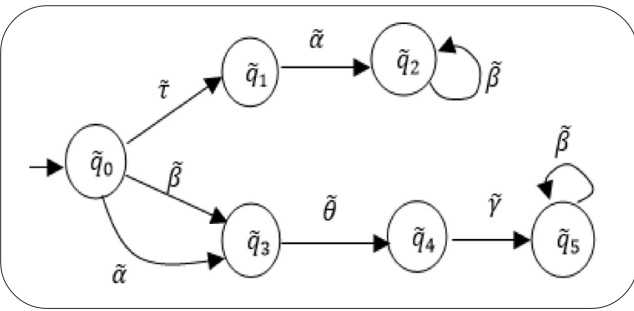

Figure 1. Fuzzy Discrte-event systems $\widetilde{G}$

\section{Verification Approach}

\subsection{Diagnoser-based Approach}

The diagnoser based approach for fuzzy predictability is based on a discrete-event structure named the "diagnoser" witch is presented for the first time in [6] to check diagnosability in crisp DESs. The diagnoser is a finite states machine (FSM) built for the system with respect to a projection onto the set of observable events and to a given fault. In this section, we adapt the diagnoser-based approach to verify the fuzzy predictability by constructing a fuzzy version of the diagnoser (we continue to use simply the name diagnoser for this fuzzy version) from a given FDES with a single failure type.

\subsection{Constructing the Diagnoser}

Let $\widetilde{\mathrm{G}}=\left(\widetilde{\mathrm{Q}}, \widetilde{\mathrm{E}}, \tilde{\delta}, \tilde{\mathrm{q}}_{0}\right)$ be a FDES. We denote by $\widetilde{\mathrm{G}}_{\mathrm{d}}$ the diagnoser built for the FDES $\widetilde{\mathrm{G}}$ and a fuzzy event $\widetilde{\sigma} \in \widetilde{\mathrm{E}}_{\mathrm{f}}$. The diagnoser is of the form $\widetilde{\mathrm{G}}_{\mathrm{d}}=\left(\widetilde{\mathrm{Q}}_{\mathrm{d}}, \widetilde{\mathrm{E}}_{\mathrm{d}}, \widetilde{\delta}_{\mathrm{d}}, \widetilde{\mathrm{q}}_{\mathrm{d} 0}\right)$ where:

$\widetilde{Q}_{d}$ is the set of the diagnoser states. $\widetilde{Q}_{d}$ is a subset of $2^{\widetilde{\mathrm{Q}} \times\left\{\mathrm{F}, \mathrm{N}^{\mu}\right\}}$. A state $\widetilde{\mathrm{q}}_{d} \in \widetilde{\mathrm{Q}}_{\mathrm{d}}$ is of a form $\widetilde{\mathrm{q}}_{d}=\left\{\left(\widetilde{\mathrm{q}}_{1}, \mathrm{l}_{1}\right),\left(\widetilde{\mathrm{q}}_{2}, \mathrm{l}_{2}\right)\right.$, $\left.\ldots,\left(\tilde{q}_{n}, l_{n}\right)\right\}$, where $\tilde{\mathrm{q}}_{\mathrm{i}} \in \widetilde{\mathrm{Q}}$ and $\mathrm{l}_{\mathrm{i}} \in\left\{\mathrm{F}, \mathrm{N}^{\mu}\right\}$ for $\mathrm{i}=1,2, \ldots, \mathrm{n}$. Label $F$ is to be interpreted as: event $\widetilde{\sigma}$ has occurred and his possibility of failure occurring exceeds the specified degree $\tilde{\Sigma}_{\mathrm{f}}(\widetilde{\sigma})$ and the system is in "failed"• state, while the label $\mathrm{N}^{\mu}$ such that $\mu \in\left\{\tilde{\Sigma}_{\mathrm{f}}(\widetilde{a}): \tilde{a} \in \widetilde{\mathrm{E}}\right\}$ and $\mu<\tilde{\Sigma}_{\mathrm{f}}(\widetilde{\sigma})$ is to be interpreted as an event $\widetilde{\sigma}$ has occurred and his possibility of failure occurring is $\mu$ but it does not exceed the specified degree, so the system is still in "normal" state.

$\tilde{q}_{d 0}$ is the initial state. It has the form $\tilde{q}_{d 0}=\left\{\left(\tilde{q}_{0}, N^{0}\right)\right\}$ which means that $\tilde{G}_{d}$ starts with a normal state.

$\tilde{E}_{d}$ is the set of events which is composed of events whose observability degree is the largest or larger than $\tilde{\Sigma}_{\mathrm{o}}(\widetilde{\sigma})$ : $\widetilde{\mathrm{E}}_{\mathrm{d}}=\widetilde{\mathrm{E}}_{\mathrm{mo}} \cup\left\{\tilde{\mathrm{a}} \in \widetilde{\mathrm{E}}: \widetilde{\Sigma}_{\mathrm{o}}(\widetilde{\mathrm{a}})>\widetilde{\Sigma}_{\mathrm{o}}(\widetilde{\sigma})\right\}$. $\widetilde{\delta_{d}}: \widetilde{Q_{d}} \times \widetilde{E_{d}} \rightarrow \widetilde{E_{d}}$ is the transition function. Let $\tilde{q}_{d}$ and $\tilde{q}_{d}{ }^{\prime}$ be two diagnoser states in $\widetilde{Q}_{d}$ such that $\tilde{q}_{d}{ }^{\prime}$ is reached from $\tilde{q}_{d}$ by $\widetilde{a} \in \widetilde{E}_{d}$, i.e., $\widetilde{q}_{d}{ }^{\prime}=\widetilde{\delta}_{d}\left(\widetilde{q}_{d}, \widetilde{a}\right)$ is defined.

Let $\widetilde{q}_{d}=\left\{\left(\widetilde{q}_{1}, l_{1}\right),\left(\widetilde{q}_{2}, l_{2}\right), \ldots,\left(\widetilde{q}_{m}, l_{m}\right)\right\}$ and $\widetilde{q}_{d}{ }^{\prime}=\left\{\left(\widetilde{q}^{\prime}{ }_{1}, l^{\prime}{ }_{1}\right),\left(\widetilde{q}_{2}^{\prime}\right.\right.$, $\left.\left.l^{\prime}{ }_{2}\right), \ldots,\left(\widetilde{q}_{n}^{\prime}, l_{n}^{\prime}\right)\right\}$. For all $i \in\{1,2, \ldots n\}$, there exists $j \in\{1$, $2, \ldots m\}$ such that $\widetilde{q}_{i}=\tilde{\delta}\left(\widetilde{q}_{j}, \widetilde{s}\right)$, where $\tilde{s}=\tilde{u} \tilde{a}, \tilde{a} \in \widetilde{E}_{d}$ and $\tilde{u} \in\left\{\tilde{v} \in \widetilde{E}^{*}: \tilde{\Sigma}_{\mathrm{o}}(\tilde{v})<\tilde{\Sigma}_{\mathrm{o}}(\widetilde{\sigma})\right\}$ and the label propagation is defined as

$$
l_{i}^{\prime}=\left(\begin{array}{lllll}
F & \text { if } & l_{j}=F \quad \text { or } \quad \tilde{\Sigma}_{f}(\tilde{S}) \geq \tilde{\Sigma}_{f}(\tilde{\sigma}) \\
N^{\tilde{\Sigma}_{f}(\tilde{s})} & \text { if } & l_{j}=N^{\mu}, \tilde{\Sigma}_{f}(\tilde{S})<\tilde{\Sigma}_{f}(\tilde{\sigma}) \quad \text { and } & \tilde{\Sigma}_{f}(\tilde{S})>\mu \quad(7) \\
N^{\mu} & \text { if } & l_{j}=N^{\mu}, \tilde{\Sigma}_{f}(\tilde{S})<\tilde{\Sigma}_{f}(\tilde{\sigma}) \quad \text { and } \quad \tilde{\Sigma}_{f}(\tilde{s})<\mu
\end{array}\right.
$$

We say that a diagnoser state $\widetilde{q}_{d}=\left\{\left(\widetilde{q}_{1}, l_{1}\right),\left(\tilde{q}_{2}, l_{2}\right), \ldots,\left(\widetilde{q}_{m}, l_{m}\right)\right\}$ is: normal if $l_{j}=N^{\mu}$ for all $j \in\{1,2, \ldots m\}$; certain if $l_{j}=F$ for all $j \in\{1,2, \ldots m\}$; and uncertain if there exist $l_{j}=N^{\mu}$ and $l_{j}=F$ or vice versa.

We denote by $\widetilde{Q}^{N}{ }_{d}$ the set of diagnoser states that are normal, by $\widetilde{Q}_{d}^{c}$ the set of states that are certain, and by $\widetilde{Q}_{d}^{U}$ the set of states that are uncertain.

Example 1 (Cont) The diagnoser $\widetilde{G}_{d}$ built for the FDES $\widetilde{G}$ depicted in Figure 1 with respect to the fuzzy event $\tilde{\theta}$ is shown in Figure 3. The diagnoser state $\left\{\left(\widetilde{q}_{5}, F\right)\right\}$ is a certain state while $\left\{\left(\widetilde{q}_{2}, N^{0}\right),\left(\widetilde{q}_{3}, N^{0}\right)\right\}$ is a normal state.

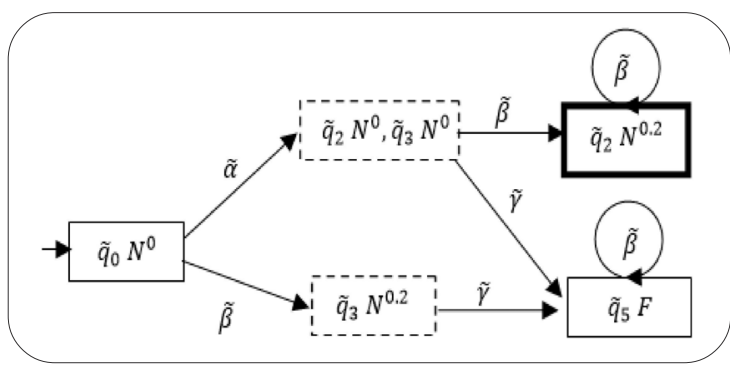

Figure 2. Diagnoser $\widetilde{G}_{d}$ with respect to $\widetilde{\theta}$ of FDES $\widetilde{G}$ example

\section{Necessary and Sufficient Condition for Fault Pre- diction in Fdess Using Diagnoser}

In this section, we give some definitions related to the diagnoser and we give the necessary and sufficient conditions of the fault prediction in FDESs using the diagnoser approach.

Definition 3 let $\widetilde{G}=\left(\widetilde{Q}, \widetilde{E}, \widetilde{\delta}, \widetilde{q}_{0}\right)$ be a FDES, we say that a set of states $\left\{\widetilde{q}_{1}, \widetilde{q}_{2}, \ldots, \widetilde{q}_{n}\right\} \subseteq \widetilde{Q}$ and a string $\left\{\widetilde{a}_{1}, \widetilde{a}_{2}, \ldots, \widetilde{a}_{n}\right\} \in$ $\widetilde{E}^{*}$ form a cycle if $\tilde{q}_{i+1}=\tilde{\delta}\left(\widetilde{q}_{i}, \tilde{a}_{i}\right)$, for $i=1,2, \ldots, n-1$ and $\tilde{q}_{1}=\widetilde{\delta}$ $\left(\tilde{q}_{n}, \tilde{a}_{n}\right)$.

The accessible part of the diagnoser from a given state is defined as follows: 
Definition 4 [7] let $\widetilde{G}=\left(\widetilde{Q}, \widetilde{E}, \widetilde{\delta}, \tilde{q}_{0}\right)$ be a FDES and $\tilde{q} \in \widetilde{Q}$. The accessible part of $\widetilde{G}$ with respect to $\widetilde{q}$ is denoted by $\operatorname{Ac}(\widetilde{G}, \widetilde{q})$ such that:

$$
A c(\tilde{G}, \widetilde{q})=\left(\tilde{Q}_{A c}, \widetilde{E}, \widetilde{\delta}_{A c}, \tilde{q}_{0}\right)
$$

Where $\tilde{Q}_{A c}=\left\{\tilde{q}^{\prime} \in \tilde{Q} \otimes \exists \tilde{s} \in \tilde{E}^{*}\right)$ s.t. $\tilde{\delta}(\tilde{q}, \tilde{s})=\tilde{q}^{\prime}$ is defined $\}$ and $\tilde{\delta}_{A c}=\left.\tilde{\delta}\right|_{\tilde{Q}_{A c} \times \tilde{E} \rightarrow \tilde{Q}_{A c}}$. This refers to the restriction of the transition function to the subset $\widetilde{Q}_{A c}$ of states.

We distinguish the set of normal states that are immediately followed by some non-normal states:

Definition 5 [7] Let $F_{D}$ be the set of the normal diagnoser states that possess an immediate successor that is not normal.

$$
\begin{aligned}
& F_{D}=\left\{x_{d} \in \widetilde{Q}_{d}^{N}: \exists y_{d}=\right. \\
& \tilde{\delta}_{d}\left(x_{d}, \tilde{a}_{o}\right) \text { is defined for } \tilde{\Sigma}_{o}\left(\tilde{a}_{o}\right)>\tilde{\Sigma}_{o}(\widetilde{\sigma}) \text { and } y_{d} \notin \widetilde{Q}_{d}^{N\}}
\end{aligned}
$$

Finally, we define the different kinds of cycles in the diagnoser as follows:

Definition 6 let $\widetilde{G}_{d}=\left(\widetilde{Q}_{d} \widetilde{E}_{d}, \widetilde{\delta}_{d}, \widetilde{q}_{d 0}\right)$ be the diagnoser of $\widetilde{G}$ with respect to $\tilde{\sigma}$. a set $\left\{\widetilde{q}_{d, 1}, \tilde{a}_{1}, \widetilde{q}_{d, 2}, \tilde{a}_{2}, \ldots, \tilde{q}_{d, k}, \tilde{a}_{k}, \widetilde{q}_{d, 1}\right\}$ is said to form:

- $\mu$ - normal cycle in $\widetilde{G}_{d}$ if $\left\{\tilde{q}_{d, 1}, \widetilde{q}_{d, 2}, \ldots, \widetilde{q}_{d, k}\right\}$ are normal states, and the set $\left\{\widetilde{q}_{d, 1}, \tilde{a}_{1}, \tilde{q}_{d, 2}, \tilde{a}_{2}, \ldots, \tilde{q}_{d, k}, \widetilde{a}_{k}, \tilde{q}_{d, 1}\right\}$ form a cycle in $\widetilde{G}_{d}$ and $\mu<\tilde{\Sigma}_{f}(\widetilde{\sigma})$.

- certain cycle in $\widetilde{G}_{d}$ if $\left\{\widetilde{q}_{d, 1}, \widetilde{q}_{d, 2}, \ldots, \widetilde{q}_{d, k}\right\}$ are certain states, and the set $\left\{\tilde{q}_{d, 1}, \tilde{a}_{1}, \tilde{q}_{d, 2}, \tilde{a}_{2}, \ldots, \tilde{q}_{d, k}, \tilde{a}_{k}, \tilde{q}_{d, 1}\right\}$ form a cycle in $\widetilde{G}_{d^{*}}$

$\cdot \mu-$ certain cycle in $\widetilde{G}_{d}$ if $\left\{\widetilde{q}_{d, 1}, \widetilde{q}_{d, 2}, \ldots, \tilde{q}_{d, k}\right\}$ are uncertain states, and the set $\left\{\widetilde{q}_{d, 1}, \tilde{a}_{1}, \widetilde{q}_{d, 2}, \tilde{a}_{2}, \ldots, \widetilde{q}_{d, k}, \tilde{a}_{k}, \tilde{q}_{d, 1}\right\}$ form a cycle in $\widetilde{G}_{d}$ and $\mu<\widetilde{\Sigma}_{f}(\widetilde{\sigma})$.

The following lemmas are useful in establishing the result that gives the necessary and sufficient condition for predictability in FDES. Lemma 1 (resp. 2, 3) is already used in [3] (resp. in [7]) while lemma 4 is introduced here to characterize normal and uncertain states in a diagnoser constructed from a FDES.

Lemma 1 [7] Let $\widetilde{G}_{d}=\left(\widetilde{Q}_{d}, \widetilde{E}_{d}, \widetilde{\delta}_{d}, \widetilde{q}_{d 0}\right)$ be the diagnoser of $\widetilde{G}$ with respect to $\tilde{\sigma} \in \widetilde{E}_{f}$. If the set of states forms a cycle in $\widetilde{G}_{d}$, then all states in the cycle have the same failure label.

Proof. It results from the fault label propagation in the diagnoser.

Lemma 2 [7] states that if there is a cycle in the diagnoser that is formed by uncertain or normal states, then there exists a corresponding cycle in the FDES $\widetilde{G}$ such that all the states in the cycle have normal labels in the cycle in $\widetilde{G}_{d}$.

Proof. The proof is given in [7]

Lemma 3 [7] states that any uncertain or certain diagnoser state is reached from a diagnoser state in $F_{D}$.

Proof. The proof is done by induction on the sequence of observable events.

Lemma 4 let $\widetilde{G}=\left(\tilde{Q}, \widetilde{E}, \widetilde{\delta}, \tilde{q}_{0}\right)$ be a FDES and $\widetilde{G}_{d}=\left(\widetilde{Q}_{d^{\prime}} \widetilde{E}_{d}\right.$, $\left.\widetilde{\delta_{d}}, \tilde{q}_{d 0}\right)$ its diagnoser. let $\mu<\tilde{\Sigma}_{f}(\widetilde{\sigma})$ and $\tilde{q}_{d} \in F_{D}$.

There is a cycle $C$ in $A c\left(\widetilde{G}_{d}, \widetilde{q}_{d}\right)$ which is normal or uncertain such that each state in $C$ contains a state of $\widetilde{G}$ labelled by if and only if the condition $Q$ holds:

$$
\begin{gathered}
Q:\left(\exists n_{0} \in \mathbb{N}\right)\left(\forall n>n_{0}\right)\left(\exists \tilde{s} \in \widetilde{\Psi}_{\widetilde{\sigma}}\left(\tilde{\Sigma}_{f}\right)\left(\exists \tilde{u} \in L_{\tilde{G}}\right)(\exists \tilde{v} \in L / \tilde{u}):\right. \\
\widetilde{\Pi}_{\tilde{\sigma}}(\tilde{u})=\widetilde{\Pi}_{\tilde{\sigma}}(\operatorname{MaxPref}(\tilde{s})) \text { and } \tilde{\Sigma}_{f}(\tilde{u})<\tilde{\Sigma}_{f}(\tilde{\sigma}) \text { and }\|\tilde{v}\| \\
=n \operatorname{and} \widetilde{\Sigma}_{f}(\tilde{u} \tilde{v})=\mu
\end{gathered}
$$

\section{Proof.}

$(\Rightarrow)$ suppose $\widetilde{q}_{d} \in F_{D}$ and $C$ is a cycle in $A c\left(\widetilde{G}_{d} \in \widetilde{q}_{D}\right)$ such that $C$ is normal or uncertain and each state in $C$ contains a state of $G$ labelled by $N^{\mu}$.

Suppose $C$ formed by the states $\tilde{x}_{d, 1}, \widetilde{x}_{d, 2}, \ldots, \tilde{x}_{d, m}$ and the observable events.

$\tilde{\sigma}_{0,1}, \tilde{\sigma}_{0,2}, \ldots, \tilde{\sigma}_{0, m}$ i.e. $\tilde{\delta}_{d}\left(\widetilde{x}_{d, i}, \tilde{\sigma}_{0, i}\right)=\tilde{x}_{d, i+1}$ for $1 \leq i \leq m$ and $\tilde{\delta}_{d}$ $\left(\tilde{x}_{d, m}, \tilde{\sigma}_{0, m}\right)=\tilde{x}_{d, 1}$

Each state $\tilde{x}_{d, i}$ contains a state of $\widetilde{G}\left(\right.$ say $\left.\tilde{x}_{i}\right)$ labelled by $N^{\mu}$ in $\widetilde{G}_{d}$ i.e. $\left(\tilde{x}_{i}, N^{\mu}\right) \in \tilde{x}_{d, i}$

From lemma 2 , there is a cycle in $\widetilde{G}$ involving the states $\tilde{x}_{1}, \widetilde{x}_{2}, \ldots, \widetilde{x}_{m}$ and $\widetilde{w}_{1}, \widetilde{w}_{2}, \ldots, \widetilde{w}_{m} \in \widetilde{E}^{*}$ with $\widetilde{\Pi}_{\sigma}\left(\widetilde{w}_{i}\right)=\widetilde{\sigma}_{0, i}$ for $i \in\{1$, $2, \ldots, m\}, \widetilde{\delta}\left(\tilde{x}_{i}, \tilde{w}_{i}\right)=x_{i+1}$ for $1 \leq i \leq m$ and $\widetilde{\delta}\left(\tilde{x}_{m}, \tilde{\sigma}_{m}\right)=\tilde{x}_{1}$.

Suppose that $\widetilde{q}_{d}$ is reached from $\widetilde{q}_{d 0}$ by $\widetilde{s}_{o} \in \widetilde{E}_{d}{ }^{*}$. So there is $\widetilde{s} \in \widetilde{\Psi}_{\widetilde{\sigma}}\left(\widetilde{\Sigma}_{f}\right)$ such that $\left.\widetilde{\Pi}_{\widetilde{\sigma}} \widetilde{s}_{i}\right)=\widetilde{s}_{o}$. take a state from $C$. without lost of generality take the state $\tilde{x}_{d 1}$. let $\tilde{t}=$ $\operatorname{Max} \operatorname{Pref}(\widetilde{s})$. we have $\widetilde{\Pi}_{\widetilde{\sigma}}(\widetilde{t})=\widetilde{s}_{o}$.

Suppose that $\tilde{x}_{d, 1}$ is reached from $\tilde{q}_{d}$ by $\tilde{s}_{o}^{\prime} \in \widetilde{E}_{d}{ }^{*}: x_{d 1}=\widetilde{\delta}_{d}$ $\left(\tilde{x}_{d, 0}, \widetilde{s}_{o} \widetilde{s}_{o}^{\prime}\right)$

Since $\left(\tilde{x}_{i}, \widetilde{N}^{\mu}\right) \in \tilde{x}_{d, i}$ for $i \in\{1,2, \ldots, m\}$ there is $\tilde{u} \in L_{\widetilde{G}}$ and $\widetilde{u}^{\prime} \in L_{\widetilde{G}} \widetilde{u}$ such that, $\left.\widetilde{\Pi}_{\tilde{\sigma}}(\widetilde{u})=\widetilde{\Pi}_{\widetilde{\sigma}} \widetilde{t}\right)=s_{o^{\prime}}, \widetilde{\Pi}_{\tilde{\sigma}}\left(\widetilde{u}^{\prime}\right)=\widetilde{s}_{o}$ and $\tilde{\delta}\left(q_{o^{\prime}}\right.$ $\left.\tilde{u} \widetilde{u}^{\prime}\right)=\tilde{x}_{1}$, moreover we have: $\forall k \geq 1, x_{1}=\delta\left(q_{o^{\prime}}, \tilde{u} \widetilde{u}^{\prime}\left(\tilde{w}_{1} \ldots \tilde{w}_{m}\right)^{k}\right)$ and $\tilde{\Sigma}_{f}\left(\tilde{u} \tilde{u}^{\prime}\left(\tilde{w}_{1} \ldots \tilde{w}_{m}\right)^{k}\right)=\mu$.

Take $n_{0}=\left\|\tilde{u}^{\prime}\right\|+k$ and $\left.\tilde{v}=\tilde{u}^{\prime}\left(\widetilde{w}_{1} \ldots \widetilde{w}_{m}\right)^{k}\right)$ then $\left(\exists n_{0} \in \mathbb{N}\right)$ 
$\left(\forall n>n_{0}\right)\left(\exists \tilde{s} \in \widetilde{\Psi}_{\tilde{\sigma}}\left(\tilde{\Sigma}_{f}\right)\left(\exists \tilde{u} \in L_{\tilde{G}}\right)(\exists \tilde{v} \in L / \tilde{u}):\right.$

$$
\begin{aligned}
& \widetilde{\Pi}_{\tilde{\sigma}}(\tilde{u})=\widetilde{\Pi}_{\tilde{\sigma}}(\operatorname{MaxPref}(\tilde{s})) \text { and } \tilde{\Sigma}_{f}(\tilde{u})< \\
& \tilde{\Sigma}_{f}(\tilde{\sigma}) \text { and }\|\tilde{v}\|=n \text { and } \tilde{\Sigma}_{f}(\tilde{u} \tilde{v})=\mu
\end{aligned}
$$

$(\Rightarrow)$ suppose that

$$
\begin{array}{r}
\left(\exists n_{0} \in \mathbb{N}\right)\left(\forall n>n_{0}\right)\left(\exists \tilde{s} \in \widetilde{\Psi}_{\widetilde{\sigma}}\left(\tilde{\Sigma}_{f}\right)\left(\exists \tilde{u} \in L_{\tilde{G}}\right)(\exists \tilde{v} \in L / \tilde{u}):\right. \\
\widetilde{\Pi}_{\tilde{\sigma}}(\tilde{u})=\widetilde{\Pi}_{\tilde{\sigma}}(\operatorname{MaxPref}(\tilde{s})) \text { and } \tilde{\Sigma}_{f}(\tilde{u})< \\
\tilde{\Sigma}_{f}(\tilde{\sigma}) \text { and }\|\tilde{v}\|=n \text { and } \tilde{\Sigma}_{f}(\tilde{u} \tilde{v})=\mu
\end{array}
$$

Take $\tilde{s} \in \widetilde{\Psi}_{\widetilde{\sigma}}\left(\tilde{\Sigma}_{f}\right)$ and let $\tilde{t}=\operatorname{MaxPref}(\tilde{s})$. We have $\tilde{\Sigma}_{f}(\tilde{t})<\widetilde{\Sigma}_{f}(\tilde{\sigma})$. Take $\tilde{u} \in L_{\tilde{G}}$ such that $\widetilde{\Pi}_{\widetilde{\sigma}}(\tilde{u})=\widetilde{\Pi}_{\widetilde{\sigma}}(\tilde{t})=\tilde{s}_{o}$ and $\tilde{\Sigma}_{f}(\tilde{u})<\tilde{\Sigma}_{f}(\tilde{\sigma})$. Let $\tilde{t}_{u o} \sigma_{o} \in L / \tilde{t}$ with $\tilde{\Sigma}_{o}\left(\tilde{t}_{u o}\right)<\tilde{\Sigma}_{o}(\tilde{\sigma})$ and $\tilde{\Sigma}_{o}\left(\sigma_{o}\right) \geq \tilde{\Sigma}_{o}(\tilde{\sigma})\left(\sigma_{o}\right.$ is the first observable reached after $\tilde{t})$. Put $\tilde{x}_{d}=\tilde{\delta}_{d}\left(\tilde{q}_{d 0}, \tilde{s}_{o}\right)$ if $\tilde{s}_{o} \neq \epsilon$ and $\tilde{x}_{d}=\tilde{q}_{d 0}$ if $\tilde{s}_{o}=\epsilon$.

Let $\tilde{y}_{d}=\tilde{\delta}_{d}\left(\tilde{x}_{d}, \sigma_{o}\right)$ its clear that $\tilde{y}_{d}$ contains a state $\left(y, l_{y}\right)$ where $l_{y}=F$ i.e. $\tilde{y}_{d} \in \tilde{Q}_{d}^{u} \cup \tilde{Q}_{d}^{c}$.

We distinguish two cases for $\tilde{x}_{d}$ :

Case $i: \tilde{x}_{d} \in \tilde{Q}_{d}^{N}$ (this is the only possible case if $\tilde{x}_{d}=\tilde{q}_{d 0}$ ) then $\tilde{x}_{d} \in F_{D}$.

Case ii: $\tilde{x}_{d} \notin \tilde{Q}_{d}^{N}$ but in this case, by lemma 3, there is a state $\widetilde{w}_{d} \in F_{D}$ reachable from $\tilde{q}_{d 0}$ such that $\tilde{x}_{d}$ is reachable from $\widetilde{w}_{d}$.

So, in all cases (i.e. case $\mathrm{i}$ or ii) there is a state $\tilde{q}_{d} \in F_{D}\left(\tilde{q}_{d}=\tilde{x}_{d}\right.$ or $\left.\tilde{q}_{d}=\tilde{w}_{d}\right)$ from which $\tilde{y}_{d}$ is reached from $\tilde{q}_{d}$ by $\sigma_{o}: \tilde{y}_{d}=\tilde{\delta}_{d}\left(\tilde{q}_{d}, \sigma_{o}\right)$. On the other hand $\tilde{v}$ may be chosen arbitrarly long and then has the from: $\left.\tilde{v}=\tilde{u}^{\prime}\left(\widetilde{w}_{1} \ldots \widetilde{w}_{m}\right)^{k}\right)$ for $k \geq 1$ with $\tilde{\Sigma}_{f}(\tilde{u} \tilde{v})=\mu$.

Let $\widetilde{\Pi}_{\widetilde{\sigma}}\left(\tilde{u}^{\prime}\right)=\tilde{s}^{\prime}{ }_{o}$ and $\widetilde{\Pi}_{\widetilde{\sigma}}\left(\widetilde{w}_{1} \ldots \widetilde{w}_{m}\right)=\tilde{s}_{o}^{\prime \prime}=\widetilde{o}_{1} \ldots \widetilde{o}_{l},(l \leq m)$.

We have two cases:

Case 1: $\tilde{s}_{o}^{\prime} \neq \epsilon$ then let $\tilde{x}_{d}^{\prime}=\tilde{\delta}_{d}\left(\tilde{x}_{d}, \tilde{s}_{o}^{\prime}\right)$ we have $\tilde{x}_{d}{ }^{\prime}=\tilde{\delta}_{d}\left(\tilde{x}_{d}{ }^{\prime}, \tilde{o}_{1} \ldots \tilde{o}_{l}\right)$.

Case 2: $\tilde{s}^{\prime}{ }_{o}=\epsilon$ then let $\tilde{x}_{d}{ }^{\prime}=\tilde{\delta}_{d}\left(\tilde{x}_{d}, \tilde{o}_{1}\right)$ we have $\tilde{x}_{d}{ }^{\prime}=$ $\tilde{\delta}_{d}\left(\tilde{x}_{d}{ }^{\prime}, \tilde{o}_{2} \ldots \tilde{o}_{l} \tilde{o}_{1}\right)$ in both cases: $\tilde{x}_{d}{ }^{\prime}=\tilde{\delta}_{d}\left(\tilde{q}_{d 0}, \tilde{s}_{o} \tilde{s}^{\prime}{ }_{o}\left(\tilde{o}_{1} \ldots \tilde{o}_{l}\right)^{k}\right)$, $k \geq 1$.

Since $\tilde{\Sigma}_{f}(\tilde{u} \tilde{v})=\mu$, it is clear that there is $\left(\tilde{x}_{i}, N^{\mu}\right) \in \tilde{x}_{d}{ }^{\prime}$ and $\tilde{x}_{d}{ }^{\prime}$ is involvede in cycle $C$ reached from $\tilde{q}_{d}$. From lemma 1, each state of $C$ contain a state labelled by $N^{\mu}$.

Now, the necessary and sufficient condition for -predictability is given as follows:

Theorem 1: Let $\tilde{G}=\left(\tilde{Q}, \tilde{E}, \tilde{\delta}, \tilde{q}_{0}\right)$ be a FDES and $\tilde{G}_{d}=\left(\tilde{Q}_{d}, \tilde{E}_{d}, \tilde{\delta}_{d}, \tilde{q}_{d 0}\right)$ its diagnoser. the fuzzy event $\tilde{\sigma}$ is 1 - predictable (completely predictable) in $\tilde{G}$ if and only if $P$ holds where:

$P$ : For all $\tilde{q}_{d} \in F_{D}$, there is only certain cycles in $\operatorname{Ac}\left(\tilde{G}_{d}, \tilde{q}_{d}\right)$.

\section{Proof.}

$(\Rightarrow) \tilde{\sigma}$ is 1 -predictable then we have

$\left(\exists n_{0} \in \mathbb{N}\right)\left(\forall n>n_{0}\right)\left(\forall \tilde{s} \in \widetilde{\Psi}_{\widetilde{\sigma}}\left(\widetilde{\Sigma}_{f}\right)\left(\forall \tilde{u} \in L_{\tilde{G}}\right)(\forall \tilde{v} \in L / \tilde{u}):\right.$

$\left[\widetilde{\Pi}_{\tilde{\sigma}}(\tilde{u})=\widetilde{\Pi}_{\tilde{\sigma}}(\operatorname{MaxPref}(\tilde{s}))\right.$ and $\tilde{\Sigma}_{f}(\tilde{u})<\tilde{\Sigma}_{f}(\tilde{\sigma})$ and $\left.\|\tilde{v}\|=n\right]$

$$
\Rightarrow \tilde{\Sigma}_{f}(\tilde{u} \tilde{v}) \geq \tilde{\Sigma}_{f}(\tilde{\sigma})(\star)
$$

For the sake of contradiction, suppose that $P$ does not holds. so, there is $\tilde{q}_{d} \in F_{D}$ and there is a cycle $C \in A C\left(\tilde{G}_{d}, \tilde{q}_{d}\right)$ such that $C$ is uncertain or normal. this means there is a state $\tilde{x}_{d, i} \in C$ containing $\left(\tilde{x}_{i}, N^{\mu}\right)$ for some $\tilde{x}_{i} \in \tilde{Q}$ and $\mu<1$. then from lemma 4 it follows that

$\left(\exists n_{0} \in \mathbb{N}\right)\left(\forall n>n_{0}\right)\left(\exists \tilde{s} \in \widetilde{\Psi}_{\tilde{\sigma}}\left(\tilde{\Sigma}_{f}\right)\left(\exists \tilde{u} \in L_{\tilde{G}}\right)(\forall \tilde{v} \in L / \tilde{u}):\right.$

$\widetilde{\Pi}_{\tilde{\sigma}}(\tilde{u})=\widetilde{\Pi}_{\tilde{\sigma}}(\operatorname{MaxPref}(\tilde{s}))$ and $\tilde{\Sigma}_{f}(\tilde{u})<\tilde{\Sigma}_{f}(\tilde{\sigma})$ and $\|\tilde{v}\|=n$ and $\tilde{\Sigma}_{f}(\tilde{u} \tilde{v})=\mu<\tilde{\Sigma}_{f}(\tilde{\sigma})$

this contradicts $(\star)$

$(\Leftarrow)$ suppose $\mathrm{P}$ holds: for all $\tilde{q}_{d} \in F_{D}$, there is only certain cycles in $\operatorname{Ac}\left(\tilde{G}_{d}, \tilde{q}_{d}\right)$.

For the sake of contradiction, suppose that $\tilde{G}$ is not 1predictable i.e. $\tilde{G}$ is $\lambda$-predictable for some $0 \leq \lambda<1$. from proposition 2 it follows that:

$$
\begin{gathered}
\left(\exists n_{0} \in \mathbb{N}\right)\left(\forall n>n_{0}\right)\left(\exists \tilde{s} \in \widetilde{\Psi}_{\tilde{\sigma}}\left(\widetilde{\Sigma}_{f}\right)\left(\exists \tilde{u} \in L_{\tilde{G}}\right)(\exists \tilde{v} \in L / \tilde{u}):\right. \\
\widetilde{\Pi}_{\tilde{\sigma}}(\tilde{u})=\widetilde{\Pi}_{\tilde{\sigma}}(\operatorname{MaxPref}(\tilde{s})) \text { and } \widetilde{\Sigma}_{f}(\tilde{u})<\widetilde{\Sigma}_{f}(\tilde{\sigma}) \text { and }\|\tilde{v}\|=n \\
\text { and } \widetilde{\Sigma}_{f}(\tilde{u} \tilde{v})=\lambda . \widetilde{\Sigma}_{f}(\tilde{\sigma})
\end{gathered}
$$

Let us put $\mu=\lambda . \tilde{\Sigma}_{f}(\tilde{\sigma})$. We have $\mu<\tilde{\Sigma}_{f}(\tilde{\sigma})$ from lemma 4 it follows that there is $\tilde{q}_{d} \in F_{D}$ and there is a cycle $C$ in $\operatorname{Ac}\left(\tilde{G}_{d}, \tilde{q}_{d}\right)$ which is normal or uncertain (hens not certain). This contradicts $P$.

To complete the picture, the following theorem gives the necessary and sufficient condition for $\lambda$ - predictability with $0 \leq \lambda<1$.

Theorem 2: Let $\tilde{G}=\left(\tilde{Q}, \tilde{E}, \tilde{\delta}, \tilde{q}_{0}\right)$ be a FDES and $\tilde{G}_{d}=\left(\tilde{Q}_{d}, \tilde{E}_{d}, \tilde{\delta}_{d}, \tilde{q}_{d 0}\right)$ its diagnoser. the fuzzy event $\tilde{\sigma}$ is $\lambda-$ predictable (partially predictable with the degree $\lambda$ ) in $\tilde{G}$ if and only if conditions R1 and R2 holds:

R1: There is $\tilde{q}_{d} \in F_{d}$, there is cycle $C$ in $A c\left(\tilde{G}_{d}, \tilde{q}_{d}\right)$ which is normal or uncertain.

R2: The minimal $\mu$-normal or $\underset{\widetilde{\Sigma}}{\mu}$-uncertain in $A c\left(\widetilde{G}_{d}, \tilde{q}_{d}\right)$ for all $\tilde{q}_{d} \in F_{d}$ satisfies $\mu=\lambda$. $\tilde{\Sigma}_{f}(\tilde{\sigma})$

\section{Proof.}

$(\Rightarrow) \tilde{\sigma}$ is $\lambda$-predictable in $\tilde{G}$. from proposition 5 we have: 
$(i)\left(\exists n_{0} \in \mathbb{N}\right)\left(\forall n>n_{0}\right)\left(\exists \tilde{s} \in \widetilde{\Psi}_{\widetilde{\sigma}}\left(\widetilde{\Sigma}_{f}\right)\left(\exists \tilde{u} \in L_{\tilde{G}}\right)(\exists \tilde{v} \in L / \tilde{u}):\right.$

$\widetilde{\Pi}_{\tilde{\sigma}}(\tilde{u})=\widetilde{\Pi}_{\tilde{\sigma}}(\operatorname{MaxPref}(\tilde{s}))$ and $\widetilde{\Sigma}_{f}(\tilde{u})<\widetilde{\Sigma}_{f}(\tilde{\sigma})$ and $\|\tilde{v}\|=n$ and $\tilde{\Sigma}_{f}(\tilde{u} \tilde{v})=\lambda \cdot \tilde{\Sigma}_{f}(\tilde{\sigma})$

(ii) $\left(\exists n_{0} \in \mathbb{N}\right)\left(\forall n>n_{0}\right)\left(\forall \tilde{s} \in \widetilde{\Psi}_{\widetilde{\sigma}}\left(\tilde{\Sigma}_{f}\right)\left(\forall \tilde{u} \in L_{\tilde{G}}\right)(\forall \tilde{v} \in L / \tilde{u}):\right.$ $\left[\widetilde{\Pi}_{\tilde{\sigma}}(\tilde{u})=\widetilde{\Pi}_{\tilde{\sigma}}(\operatorname{MaxPref}(\tilde{s}))\right.$ and $\tilde{\Sigma}_{f}(\tilde{u})<$

$\tilde{\Sigma}_{f}(\tilde{\sigma})$ and $\left.\|\tilde{v}\|=n\right] \Rightarrow \tilde{\Sigma}_{f}(\tilde{u} \tilde{v}) \geq \lambda \cdot \tilde{\Sigma}_{f}(\tilde{\sigma})$

Put $\lambda . \tilde{\Sigma}_{f}(\tilde{\sigma})=\mu$ then $R 1$ follows from (i) and lemma 4 .

To show $R 2$, suppose that $\mu$ is not minimal, i.e. there $\mu^{\prime}<$ $\mu$, there is $\tilde{q}_{d} \in F_{d}$ and a cycle $C$ in $A c\left(\tilde{G}_{d}, \tilde{q}_{d}\right)$ such that $C$ is $\mu^{\prime}$ - normal or $\mu^{\prime}$ - uncertain, from lemma 4 it follows that:

$$
\begin{aligned}
& \left(\exists n_{0} \in \mathbb{N}\right)\left(\forall n>n_{0}\right)\left(\exists \tilde{s} \in \widetilde{\Psi}_{\widetilde{\sigma}}\left(\widetilde{\Sigma}_{f}\right)\left(\exists \tilde{u} \in L_{\tilde{G}}\right)(\exists \tilde{v} \in L / \tilde{u}):\right. \\
& \widetilde{\Pi}_{\widetilde{\sigma}}(\tilde{u})=\widetilde{\Pi}_{\tilde{\sigma}}(\operatorname{MaxPref}(\tilde{s})) \text { and } \tilde{\Sigma}_{f}(\tilde{u})<\widetilde{\Sigma}_{f}(\tilde{\sigma}) \text { and }\|\tilde{v}\|=n \\
& \text { and } \widetilde{\Sigma}_{f}(\tilde{u} \tilde{v})=\mu^{\prime}<\lambda . \widetilde{\Sigma}_{f}(\tilde{\sigma})
\end{aligned}
$$

This contradicts the condition (ii) of $\lambda$-predictability.

$(\Leftarrow)$ Suppose that the two conditions $R 1$ and $R 2$ hold:

$\boldsymbol{R} 1$ : There is $\tilde{q}_{d} \in F_{d}$, there is cycle $C$ in $A c\left(\widetilde{G}_{d}, \tilde{q}_{d}\right)$ which is normal or uncertain.

R2: The minimal $\mu$-normal or $\mu$-uncertain in $A c\left(\widetilde{G}_{d}, \widetilde{q}_{d}\right)$ for all $\tilde{q}_{d} \in F_{d}$ satisfies $\mu=\lambda$. $\widetilde{\Sigma}_{f}(\widetilde{\sigma})$.

From $R 1$ and lemma 4 we have:

$(i)\left(\exists n_{0} \in \mathbb{N}\right)\left(\forall n>n_{0}\right)\left(\exists \tilde{s} \in \widetilde{\Psi}_{\widetilde{\sigma}}\left(\widetilde{\Sigma}_{f}\right)\left(\exists \tilde{u} \in L_{\tilde{G}}\right)(\exists \tilde{v} \in L / \tilde{u}):\right.$ $\widetilde{\Pi}_{\tilde{\sigma}}(\tilde{u})=\widetilde{\Pi}_{\tilde{\sigma}}(\operatorname{MaxPref}(\tilde{s}))$ and $\tilde{\Sigma}_{f}(\tilde{u})<\tilde{\Sigma}_{f}(\tilde{\sigma})$ and $\|\tilde{v}\|=n$ and $\tilde{\Sigma}_{f}(\tilde{u} \tilde{v})=\lambda \cdot \widetilde{\Sigma}_{f}(\tilde{\sigma})$

From $R 2$ we have:

$(i i)\left(\exists n_{0} \in \mathbb{N}\right)\left(\forall n>n_{0}\right)\left(\forall \tilde{s} \in \widetilde{\Psi}_{\widetilde{\sigma}}\left(\widetilde{\Sigma}_{f}\right)\left(\forall \tilde{u} \in L_{\tilde{G}}\right)(\forall \tilde{v} \in L / \tilde{u}):\right.$

$\left[\widetilde{\Pi}_{\tilde{\sigma}}(\tilde{u})=\widetilde{\Pi}_{\widetilde{\sigma}}(\operatorname{MaxPref}(\tilde{s}))\right.$ and $\tilde{\Sigma}_{f}(\tilde{u})<\tilde{\Sigma}_{f}(\tilde{\sigma})$ and $\left.\|\tilde{v}\|=n\right]$

$$
\Rightarrow \widetilde{\Sigma}_{f}(\tilde{u} \tilde{v}) \geq \lambda \cdot \tilde{\Sigma}_{f}(\tilde{\sigma})
$$

From (i), (ii) and from Proposition 5 we deduce that $\tilde{\sigma}$ is $\lambda$ predictable in $\widetilde{G}$.

The necessary and sufficient condition for particular case of -predictability may be simplified as follows:

Corollary 3: Let $\widetilde{G}=\left(\widetilde{Q}, \widetilde{E}, \widetilde{\delta}, \widetilde{q}_{0}\right)$ be a FDES and $\widetilde{G}_{d}=\left(\widetilde{Q}_{d^{p}}\right.$ $\left.\widetilde{E}_{d}, \widetilde{\delta}_{d}, \widetilde{q}_{d 0}\right)$ its diagnoser. The fuzzy event $\tilde{\sigma}$ is 0 -predictable (completely non predictable) in $\widetilde{G}$ if and only if there is $\widetilde{q}_{d} \in \widetilde{F}_{d}$ and 0-normal or 0-uncertian cycle in $\operatorname{Ac}\left(\widetilde{G}_{d}\right.$, $\left.\tilde{q}_{d}\right)$.

Proof. Follows immediately from theorem 1.

Example 1 (Cont) Let us take again the example of the
FDES depicted in Figure 1 and using Theorem 2 we calculate the prediction of the fuzzy event $\widetilde{\theta}$ in the FDES $\widetilde{G}$, where its diagnoser is shown in Figure 2. First, we determine from the diagnoser the set of normal states that has a certain or uncertain diagnoser state as successor the set $F_{D}=\left\{\left\{\left(\tilde{q}_{2}, N^{0}\right),\left(\tilde{q}_{3}, N^{0}\right)\right\}\left\{\left(\tilde{q}_{3}, N^{0.2}\right)\right\}\right\}$ the states of the set $F_{D}$ are presented with dashed rectangles in the diagnoser. Then $A c\left(G_{d},\left\{\left(\widetilde{q}_{2}, N^{0}\right),\left(\tilde{q}_{3}\right.\right.\right.$, $\left.\left.\tilde{N}^{0}\right)\right\}$ ) contains a single cycle that is a minimal 0.2-normal cycle and it is presented in the diagnoser with bold-border rectangle. Thus based on the theorem 2 the fuzzy event $\tilde{\theta}$ is partially predictable with a degree $\lambda=\mu / \widetilde{\Sigma}_{f}(\widetilde{\sigma})=0.2 / 0.7$ $=0.28$.

\section{Illustrative Examples}

In this section, we give some examples to illustrate the efficiency of the proposed approach using the diagnoser.

\section{Example 1: A completely predictable FDES}

In this example we consider a FDES $\widetilde{G}_{2}=\left(\widetilde{Q}, \widetilde{E}, \widetilde{\delta}, \widetilde{q}_{0}\right)$. We depict again this system in Figure 3 . The set $\widetilde{Q}$ of fuzzy states of $G_{2}$ is: $\tilde{Q}=\left(\tilde{q}_{1}, \tilde{q}_{2}, \tilde{q}_{3}, \tilde{q}_{4}\right)$ such that the initial state is $\tilde{q}_{1}=[0.3,0.9,0.4,0.2]$ and the other states are calculated using max-min operation given in Definition 3 . The fuzzy events set is: $\widetilde{E}=\{\widetilde{\alpha}, \widetilde{\sigma}, \widetilde{\gamma}\}$. These fuzzy events are given in the following matrices:

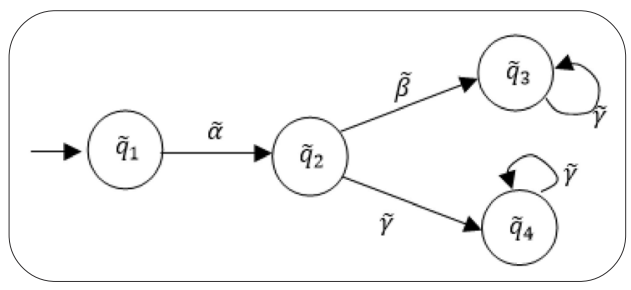

Figure 3. Example 2 FDES $\widetilde{G}_{2}$

$\tilde{\alpha}=\left(\begin{array}{llll}0.2 & 0.4 & 0.9 & 0.30 \\ 0 & 0.2 & 0.5 & 0.6 \\ 0 & 0 & 0.5 & 0.1 \\ 0 & 0 & 0 & 0.6\end{array}\right) \tilde{\beta}=\left(\begin{array}{llll}0.2 & 0 & 0 & 0 \\ 0.5 & 0.4 & 0 & 0 \\ 0.1 & 0.6 & 0.5 & 0 \\ 0.2 & 0.5 & 0.8 & 0.4\end{array}\right) \tilde{\gamma}=\left(\begin{array}{llll}0.5 & 0.6 & 0.4 & 0.2 \\ 0 & 0.1 & 0.3 & 0.8 \\ 0 & 0 & 0.6 & 0.2 \\ 0 & 0 & 0 & 0.1\end{array}\right)$

Suppose that the observability degrees of the events and their possibilities of failure are given by:

$$
\begin{aligned}
& \tilde{\Sigma}_{o}(\tilde{\alpha})=0.5, \tilde{\Sigma}_{o}(\tilde{\beta})=0.4, \tilde{\Sigma}_{o}(\tilde{\gamma})=0.6 \\
& \tilde{\Sigma}_{f}(\tilde{\alpha})=0.1, \tilde{\Sigma}_{f}(\tilde{\beta})=0.3, \tilde{\Sigma}_{f}(\tilde{\gamma})=0.4
\end{aligned}
$$

Thus, the set of faulty events is: $\widetilde{E}_{f}=\{\tilde{\alpha}, \widetilde{\beta}, \tilde{\gamma}\}$ and we want to verify the predictability of all the events of $\tilde{E}_{f}$ in $\tilde{G}_{2}$.

The diagnosers with respect to each event $\tilde{\alpha}, \widetilde{\beta}, \tilde{\gamma} \in \widetilde{E}_{f}$ in $\widetilde{G}_{2}$ are shown in Figure 4 and Figure 5.

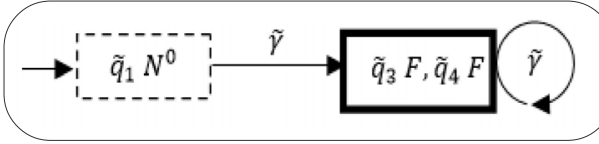

Figure 4. Diagnoser of the FDES $\widetilde{G}_{2}$ with respect to $\widetilde{\alpha}$ and $\tilde{\gamma}$ 


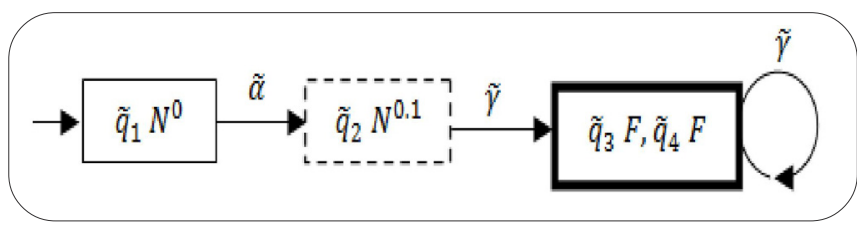

Figure 5. Diagnoser of the FDES $\widetilde{G}_{2}$ with respect to $\widetilde{\beta}$

Note that in the diagnoser with respect to $\tilde{\gamma}$, we have used the set of maximal observable events $\widetilde{E}_{m o}=\{\widetilde{\gamma}\}$, defined in section 3 in order to avoid having an empty set of events in the diagnoser.

From Fig. 4 and Fig. 5, it is clear that all cycles in the diagnosers are certain cycles. Therefore, by theorem 1 , all fuzzy events $\tilde{\alpha}, \widetilde{\beta}, \tilde{\gamma} \in \widetilde{E}_{f}$ in the FDES $\widetilde{G}_{2}$ are completely predictable, so we can say that the FDES $\widetilde{G}_{2}$ is completely predictable.

\section{Example 2: Crisp DES}

In this example, we show that we can use the approach presented in this paper to deal with crisp DESs. A crisp DES with $n$ crisp states is seen as a special case of FDES where: each crisp state is represented as a binary vector of dimension $n$ in which only one element is equal to 1 ; and each event is represented as a binary matrix of dimensions $n \times n$. Therefore, the fuzzy fault prediction approach presented above may be used to deal with the problem of fault prediction for crisp DESs.

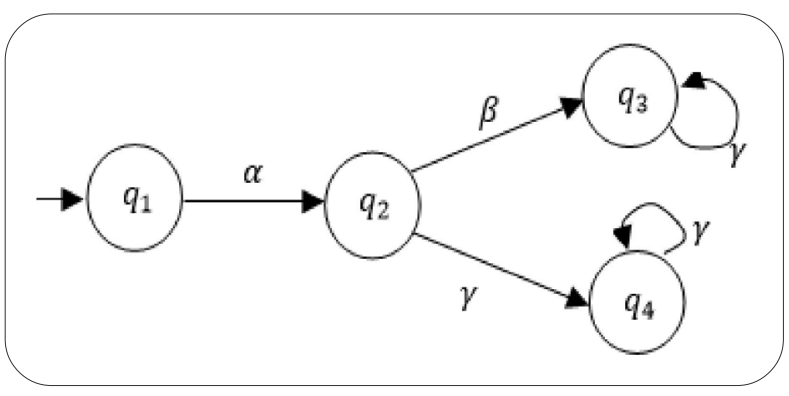

Figure 6. Example 2 Crisp DES $G_{3}$

Consider the crisp DES $G_{3}$ depicted in Figure 6 , in which the set of observable events is $E_{o}=\{\alpha, \gamma\}$ and the set of failure events is $E_{f}=\{\beta\}$. Let us show that the event $\beta$ is predictable (completely not predictable) in $G_{3}$.

The crisp DES $G_{3}$ can be viewed as a special FDES with the fuzzy states: $q_{1}=[1,0,0,0], q_{2}=[0,1,0,0], q_{3}=[0,0,1$, $0], q_{4}=[0,0,0,1]$ and the fuzzy events:

$\alpha=\left(\begin{array}{llll}0 & 1 & 0 & 0 \\ 0 & 0 & 0 & 0 \\ 0 & 0 & 0 & 0 \\ 0 & 0 & 0 & 0\end{array}\right) \beta=\left(\begin{array}{llll}0 & 0 & 0 & 0 \\ 0 & 0 & 1 & 0 \\ 0 & 0 & 0 & 0 \\ 0 & 0 & 0 & 0\end{array}\right) \gamma=\left(\begin{array}{llll}0 & 0 & 0 & 0 \\ 0 & 0 & 1 & 1 \\ 0 & 0 & 1 & 0 \\ 0 & 0 & 0 & 1\end{array}\right)$

Since $\tilde{E}_{o}=\{\alpha, \gamma\}$, we put: $\tilde{\Sigma}_{o}(\alpha)=\tilde{\Sigma}_{o}(\gamma)=1$ and
$\tilde{\Sigma}_{o}(\beta)=0$. Analogously, since $\tilde{E}_{f}=\{\beta\}$, we put: $\tilde{\Sigma}_{f}(\alpha)=\tilde{\Sigma}_{f}(\gamma)=0$ and $\tilde{\Sigma}_{f}(\beta)=1$.

Next, we verify the above result by means of the diagnoser approach. The diagnoser $G_{d}$ of $G_{3}$ with respect to $\beta$ is depicted in Figure 7.

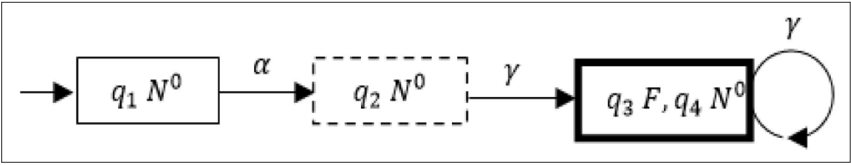

Figure 7. Diagnoser $G_{d}$ with respect to $\beta$ in example 2 Crisp $\operatorname{DES} G_{3}$

The set $F_{D}$ of normal states having a certain or an uncertain state as an immediate successor is: $F_{D}=\left\{\left(q_{2}, N^{0}\right)\right\}$. Note that the states of $F_{D}$ are presented with dashed rectangles in the diagnoser (this convention is kept for the subsequent examples). Then, clearly $A c\left(G_{d}\left\{\left(q_{2}, N^{0}\right)\right)\right.$ contains a single cycle that is a minimal 0 -uncertain cycle (it is presented in the diagnoser with a bold-border rectangle). Thus, the event $\beta$ is completely non-predictable in $G_{3}$.

\section{Example 3: Treatment Process of an Animal}

Let consider the treatment process of an animal, modeled by fuzzy DES $\widetilde{G}_{4}$ depicted in Fig. 8 (this example is inspired from [3]). This animal becoming sick with a new disease. The drugs Theophylline, Ipratropium Bromide, Erythromycin Ethylsuccinate, and Dopamine are denoted by fuzzy event $\widetilde{\alpha}, \widetilde{\beta}, \widetilde{\gamma}$ and $\widetilde{\theta}$, respectively. The doctor believes that these drugs may be useful for the disease. A state in this fuzzy DES is denoted by a vector $\tilde{q}=\left(a_{1}\right.$, $a_{2}, a_{3}$ ) which means that the animal's condition can simultaneously belong to "good", "fair" and "poor" with respective membership degrees $a_{1}, a_{2}$ and $a_{3}$. The initial state is $\tilde{q}_{0}=[0.9,0.1,0]$ and the other states calculated using max-min operation (see Definition 1) are: $\widetilde{q}_{1}=[0.4$, $0.9,0.4], \tilde{q}_{2}=[0.9,0.4,0.4], \tilde{q}_{3}=[0.4,0.9,0.4], \tilde{q}_{4}=[0.9,0.9,0.4]$, $\tilde{q}_{5}=[0.5,0.1,0], \tilde{q}_{6}=[0.5,0.4,0.4], \tilde{q}_{7}=[0.5,0.4,0.4]$.

Since it is imprecise to determine the exact point at which the animal has changed from one state to another after a drug treatment, each fuzzy event is modeled by a $3 \times 3$ matrix.

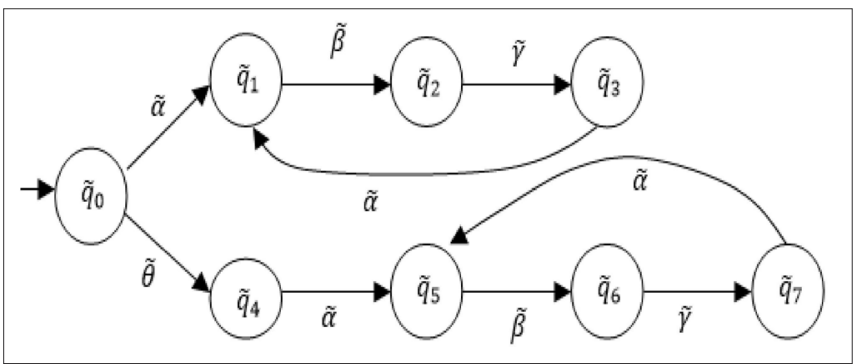

Figure 8. Treatment process [3] modeled by FDES $\widetilde{G}_{4}$ 


$$
\begin{aligned}
& \tilde{\alpha}=\left(\begin{array}{lll}
0.4 & 0.9 & 0.4 \\
0 & 0.4 & 0.4 \\
0 & 0 & 0.4
\end{array}\right) \tilde{\beta}=\left(\begin{array}{lll}
0.4 & 0 & 0 \\
0.9 & 0.4 & 0 \\
0.4 & 0.4 & 0.4
\end{array}\right) \\
& \tilde{\gamma}=\left(\begin{array}{lll}
0.9 & 0.9 & 0.4 \\
0 & 0.4 & 0.4 \\
0 & 0 & 0.4
\end{array}\right) \tilde{\theta}=\left(\begin{array}{lll}
0.5 & 0 & 0 \\
0.1 & 0.1 & 0 \\
0.1 & 0.1 & 0.1
\end{array}\right)
\end{aligned}
$$

Suppose that the observability degrees and the failure possibilities of the events are defined as follows:

$$
\begin{aligned}
& \tilde{\Sigma}_{o}(\tilde{\alpha})=0.5, \tilde{\Sigma}_{o}(\tilde{\beta})=0.4, \tilde{\Sigma}_{o}(\tilde{\gamma})=0.6, \tilde{\Sigma}_{o}(\tilde{\theta})=0.3 \\
& \tilde{\Sigma}_{f}(\tilde{\alpha})=0.1, \tilde{\Sigma}_{f}(\tilde{\beta})=0.2, \tilde{\Sigma}_{f}(\tilde{\gamma})=0.3, \tilde{\Sigma}_{f}(\tilde{\theta})=0.4 .
\end{aligned}
$$

Let us now apply the diagnoser approach. First, we construct the diagnosers for each fuzzy event in $\widetilde{E}_{f}=\{\tilde{\alpha}, \widetilde{\beta}, \tilde{\gamma}$ and $\widetilde{\theta\}}$ (see Figures 9-11).

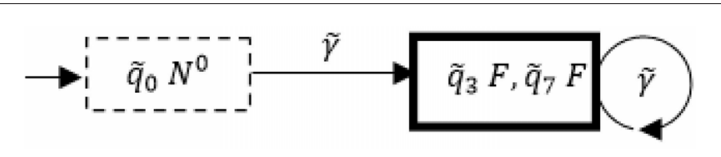

Figure 9. Diagnoser of FDES $\widetilde{G}_{4}$ with respect to $\widetilde{\alpha}, \tilde{\gamma}$

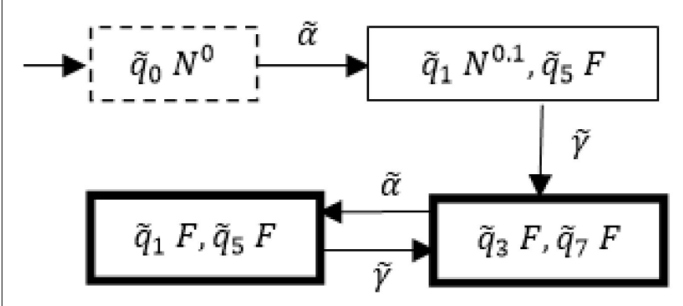

Figure 10. Diagnoser of FDES $\widetilde{G}_{4}$ with respect to $\widetilde{\beta}$

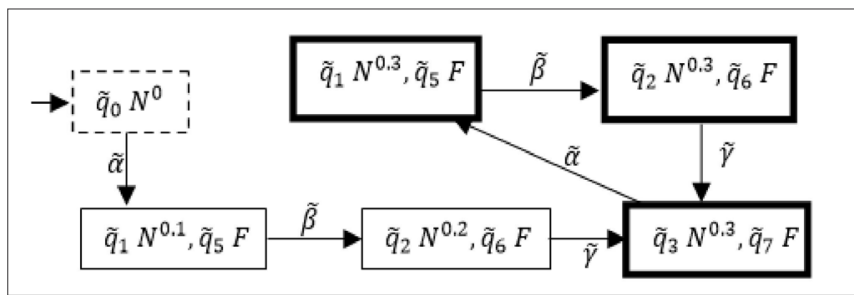

Figure 11. Diagnoser of FDES $\widetilde{G}_{4}$ with respect to $\widetilde{\theta}$

1. The diagnoser with respect to $\widetilde{\alpha}($ resp. to $\tilde{\gamma})$ is shown in Figure 9. Its set of events is: $\widetilde{E}_{d}=\{\widetilde{\gamma}\}$ and its states are: $\tilde{q}_{d 0}=\left\{\left(\tilde{q}_{0}, N^{0}\right)\right\}$ and $\tilde{q}_{d, 1}=\left\{\left(\tilde{q}_{3}, F, \tilde{q}_{7}, F\right)\right\}$. Since all cycles in the diagnoser with respect to $\tilde{\alpha}$ and $\tilde{\gamma}$ are certain, $\tilde{\alpha}$ and $\tilde{\gamma}$ are completely predictable in $\tilde{G}_{4}$.

2. The diagnoser with respect to $\tilde{\beta}$ is shown in Fig. reffig:10. Its set of event is: $\widetilde{E}_{d}=\{\tilde{\alpha}, \tilde{\gamma}\}$ and its states are: $\tilde{q}_{d 0}=\left\{\left(\tilde{q}_{0}, N^{0}\right)\right\}, \tilde{q}_{d, 1}=\left\{\left(\tilde{q}_{1}, N^{0.1}\right),\left(\tilde{q}_{5}, F\right)\right\}, \tilde{q}_{d, 2}=\left\{\left(\tilde{q}_{3}, F\right)\right.$, $\left.\left(\tilde{q}_{7}, F\right)\right\}$ and $\tilde{q}_{d, 3}=\left\{\left(\tilde{q}_{1}, F\right),\left(\tilde{q}_{5}, F\right)\right\}$.

All cycles in the diagnoser with respect to $\widetilde{\beta}$ are certain. Then, $\tilde{\beta}$ is completely predictable in $\widetilde{G}_{4}$.
3. The diagnoser with respect to $\tilde{\theta}$ is shown in Fig. 11. Its set of events is: $\widetilde{E}_{d}=\{\widetilde{\alpha}, \widetilde{\beta}, \widetilde{\gamma}\}$ and its states are:

$\tilde{q}_{d 0}=\left\{\left(\tilde{q}_{0}, N^{0}\right)\right\}, \tilde{q}_{d, 1}=\left\{\left(\tilde{q}_{1}, N^{0.1}\right),\left(\tilde{q}_{5}, F\right)\right\}$,

$\tilde{q}_{d, 2}=\left\{\left(\tilde{q}_{2}, N^{0.2}\right),\left(\tilde{q}_{6}, F\right)\right\}, \tilde{q}_{d, 3}=\left\{\left(\tilde{q}_{3}, N^{0.3}\right),\left(\tilde{q}_{7}, F\right)\right\}$,

$\tilde{q}_{d, 4}=\left\{\left(\tilde{q}_{1}, N^{0.3}\right),\left(\tilde{q}_{5}, F\right)\right\}, \tilde{q}_{d, 5}=\left\{\left(\widetilde{q}_{2}, N^{0.3}\right),\left(\tilde{q}_{6}, F\right)\right\}$.

The set $F_{D}$ of normal states that have as an immediate successor a certain or uncertain diagnoser state is $F_{D}=\left\{\left(\tilde{q}_{0}, N^{0}\right)\right\}$. Then $A c\left(G_{d},\left(\tilde{q}_{0}, N^{0}\right)\right)$ contains a minimal 0.3-uncertain cycle $\left\{\tilde{q}_{d, 3}, \tilde{\alpha}, \tilde{q}_{d, 4}, \tilde{\beta}, \tilde{q}_{d, 5}, \tilde{\gamma}, \tilde{q}_{d, 3}\right\}$ where $\tilde{q}_{d, 3}=\left\{\left(\tilde{q}_{3}, N^{0.3}\right),\left(\tilde{q}_{7}, F\right)\right\}, \tilde{q}_{d, 4}=\left\{\left(\tilde{q}_{1}, N^{0.3}\right),\left(\tilde{q}_{5}, F\right)\right\}$ and $\tilde{q}_{d, 5}=$ $\left\{\left(\tilde{q}_{2}, N^{0.3}\right),\left(\tilde{q}_{6}, F\right)\right\}$.

We have: $\mu=\lambda \tilde{\Sigma}_{f}(\tilde{\theta})$. Then, since $\mu=0.3$ and $\tilde{\Sigma}_{f}(\tilde{\theta})=0.4$, we obtain: $\lambda=0.75$. We deduce that the fuzzy event is partialy predictable in $\tilde{G}_{4}$ with degree 0.75 .

\section{Conclusion}

This paper deals with fault prediction in FDESs using the Diagnoser structure. Two theorems are formulated on the basis of some diagnoser proprieties. These theorems quantifies the fault prediction degree of a failure event at different levels and take their values in the interval $[0 ; 1]$ rather than in the binary set $\{0,1\}$ including (i) the completely prediction of a faulty or non-faulty of any event in the system (ii) the prediction with a degree of faulty event. We have also shown that the new setting generalizes the classical setting of predictability in crisp DESs. Indeed, by using an adapted presentation of any crisp DES as a special fuzzy DES, our proposed fuzzy approach leads to the correct decision about the (classical) predictability of any failure event in the crisp DES.

Further issues regarding the faulty pattern prediction will be considered in future work. The first perspective is to extend our approach to deal with systems where the fault is a pattern not a single fault. An additional aspect that we aim to consider is to bring into play abstraction techniques to improve the construction of the diagnoser and help tackling the inherent combinatorial explosion problem.

\section{References}

[1] Zadeh, L. A. (1996). Fuzzy logic computing with words, IEEE Transactions on Fuzzy Systems, 4 (2) (May), 103111.

[2] Cassandras, C. G., Lafortune, S. (1999). Introduction to Discrete Event Systems. Boston, MA: Kluwer.

[3] Liu, F., Qiu, D. W. (2009). Diagnosability of fuzzy discrete-event systems: a fuzzy approach, IEEE Transactions on Fuzzy Systems, 17 (2) 372-384

[4] Qiu, D. W., Liu, F. C. (2009). Fuzzy discrete event 
systems under fuzzy observability and a test-algorithm. IEEE Trans Fuzzy Syst, 17, 578-589,

[5] Qiu, D. (2005). Supervisory control of fuzzy discrete event systems: A formal approach, IEEE Transactions on Systems, Man, Cybern. B, Cybern., 35 (1) (February) $72-88$.
[6] Sampath, M., Sengupta, R., Lafortune, S., Sinnamohideen, K., Teneketzis, D. (1995). Diagnosability of discrete event systems. IEEE Transactions on Automatic Control, 40 (9) 1555-1575.

[7] Genc, S., Lafortune, S. (2009). Predictability of event occurrences in partially-observed discrete-event systems. Automatica, 45 (2) 301- 311. 